\title{
亶 \\ Balanço hídrico e excreção renal de metabólitos em cabras leiteiras alimentadas com feno de alfafa e palma forrageira
}

\author{
[Water balance and renal excretion of metabolites in dairy goats \\ fed alfalfa hay and spineless cactus]
}

\section{"Artigo Científico/Scientific Article"}

\section{Levi Auto Lopes ${ }^{1 *}$, Francisco Fernando Ramos de Carvalho ${ }^{1}$, Ana Maria Duarte Cabral Belo ${ }^{2}$, Ângela Maria Vieira Batista ${ }^{1}$, Karine Silva Camargo ${ }^{1}$, José Ricardo Coelho da Silva ${ }^{2}$, Daniel Barros Cardoso ${ }^{1}$, Cleyton Charles Dantas Carvalho ${ }^{3}$}

\footnotetext{
${ }^{1}$ Departamento de Zootecnia, Universidade Federal Rural de Pernambuco (UFRPE), Recife-PE, Brasil.

${ }^{2}$ Departamento de Zootecnia, Unidade Serra Talhada, Universidade Federal Rural de Pernambuco (UFRPE), Serra Talhada-PE, Brasil.

${ }^{3}$ Departamento de Medicina Veterinária, Universidade Federal Rural de Pernambuco (UFRPE), Recife-PE, Brasil.

*Autor para correspondência/Corresponding author: E-mail: levi_auto@hotmail.com
}

\section{Resumo}

Objetivou-se determinar os efeitos da substituição do feno de Tifton (Cynodon spp.) por feno de alfafa (Medicago Sativa) em dietas à base de palma forrageira (Nopalea cochenillifera) sobre o balanço hídrico, funções renais e excreção mineral de cabras leiteiras. Utilizou-se 12 cabras da raça Saanen, com peso vivo de $46,23 \pm 7,5 \mathrm{~kg}$, produção de $3,0 \mathrm{~kg}$ leite/dia. O delineamento experimental foi quadrado latino $(4 \times 4)$, com quatro tratamentos $(0 \% ; 33,3 \% ; 66,7 \%$ e $100 \%$ de substituição) e quatro períodos para coleta de dados e amostras. O consumo de matéria seca (CMS), expresso em $\mathrm{kg}$ /dia e consumo de água total apresentaram aumento linear $(p<0,05)$ em função da substituição do feno de Tifton pelo feno de alfafa, variando de 2,25 a 2,49 kg/dia e de 10,4 a 11,6 L/dia, para os níveis 0 a 100\%, respectivamente. As perdas de água, taxa de depuração endógena de creatinina (TDECr), taxa de excreção de urina (TEU), Ca, $\mathrm{P}, \mathrm{Mg}$ e ureia no plasma e urina, assim como índice de excreção urinária (IEUr) e taxas de excreção fracionadas (TEF) de cabras alimentadas com feno de alfafa em substituição ao feno de Tifton em dietas à base de palma forrageira, não foram influenciados $(\mathrm{P}>0,05)$ pelas dietas. A substituição do feno de Tifton por feno de alfafa em até $300 \mathrm{~g} / \mathrm{kg}$ de matéria seca, em dietas à base de palma forrageira para cabras em lactação, influencia no consumo de água, sem alterar os índices de excreção urinária.

Palavras-chave: água; cactáceas; caprinos; minerais.

\begin{abstract}
This study evaluates the effects of replacing Tifton hay (Cynodon spp.) with alfalfa hay (Medicago Sativa) in spineless cactus-based diets (Nopalea cochenillifera) on water balance, renal function, and mineral excretion of dairy goats. For that purpose, twelve Saanen goats with a live weight of $46.23 \pm 7.5 \mathrm{~kg}$ and production of $3.0 \mathrm{~kg}$ milk/day were used. The experimental design was a Latin square $(4 \mathrm{x} 4)$ with four treatments $(0 \% ; 33.3 \%$; $66.7 \%$; and $100 \%$ replacement) and four periods for data collection and sampling. Dry matter intake (DMI), expressed in $\mathrm{kg} / \mathrm{day}$, and total water intake showed a linear increase $(\mathrm{p}<0.05)$ as a function of replacing Tifton hay with alfalfa hay, ranging from 2.25 to $2.49 \mathrm{~kg} /$ day and from 10.4 to $11.6 \mathrm{~L} /$ day, at 0 to $100 \%$ replacement, respectively. Water loss; endogenous creatinine clearance rate (ECCR); $\mathrm{Ca}, \mathrm{P}, \mathrm{Mg}$, and urea in the plasma and urine; as well as urinary excretion rate (UER) and the fractional excretion rates (FER) of goats fed alfalfa hay instead of Tifton hay in spineless cactus-based diets were not influenced $(\mathrm{P}>0.05)$ by treatments. Replacing Tifton hay with alfalfa hay up to $300 \mathrm{~g} / \mathrm{kg}$ dry matter in spineless cactus-based diets for lactating goats influences water consumption without altering urinary excretion rates.
\end{abstract}

Keywords: water; cactaceae; goats; minerals. 


\section{Introdução}

Nos sistemas de produção animal, a qualidade dos alimentos que compõem a dieta é imprescindível na busca por eficiência produtiva. Para atender às exigências nutricionais dos animais, a utilização do volumoso de melhor qualidade objetiva a redução da utilização de concentrados, mantendo o fornecimento de proteína verdadeira e energia para o animal, contudo, sem comprometer o desempenho e obter diminuição do custo.

A palma forrageira associada com leguminosas podem trazer diversos benefícios nutricionais, visto que são forrageiras com alto valor nutritivo, como a alfafa (Medicago sativa), que além de ser rica em energia e proteína bruta, apresenta alta concentração de cálcio e fósforo.

Assim como Batista et al. (2009) relataram alta concentração de $\mathrm{Ca}$ seguido de $\mathrm{Mg}$ e $\mathrm{K}$ na palma forrageira. Vieira et al. (2008) descreveram que devido sua composição, o consumo de palma forrageira pode resultar em efeitos diuréticos do trato renal em caprinos, como também modificar as funções renais e excreção mineral. É notório que pouco se sabe a respeito do alto percentual de elementos minerais e de sua real interação e disponibilidade, uma vez que os minerais se interrelacionam de forma sinérgica ou antagônica (Neto et al., 2016). Tornam-se necessários mais pesquisas neste âmbito, visto que, a urina está entre as principais vias de excreção do organismo e as excreções renais podem oferecer informações do estado fisiológico do animal, sob desafios nutricionais e desordens metabólicas de pequenos ruminantes.

Assim, a hipótese que a substituição do feno de uma gramínea como o Tifton por leguminosa alfafa em dietas à base de palma forrageira, poderia ser utilizada sem causar prejuízos na eficiência no uso da água e excreção renal de cabras. Objetivouse, com o presente estudo, determinar os efeitos da substituição do feno de Tifton pelo feno de alfafa em dietas associadas à palma forrageira, sobre o balanço hídrico, funções renais e excreção mineral de cabras leiteiras.

\section{Material e Métodos}

A pesquisa foi realizada no setor de caprinos do Departamento de Zootecnia da Universidade Federal Rural de Pernambuco (UFRPE) em Recife, Brasil.

\section{Animais e manejo experimental}

Foram utilizadas 12 cabras da raça Saanen, com peso vivo médio de $46,23 \pm 7,5 \mathrm{~kg}$, média de produção de $3,0 \mathrm{~kg}$ leite/dia, todas as cabras de terceira e quarta lactações, sincronizadas a obter todos os partos na mesma semana e garantir a homogeneidade no período de lactação. Os animais foram vacinados, tratados contra endoparasitas, identificados e distribuídos ao acaso, em baias de madeira individuais e suspensas, medindo $1,10 \times$ $1,20 \mathrm{~m}$, providas de comedouro e bebedouro. Foram realizadas duas ordenhas diárias, às 07 e 15 horas, sendo a produção de leite de cada animal registrada durante todo o período experimental.

$\mathrm{O}$ delineamento experimental foi quadrado latino (4x4), com quatro animais, quatro períodos experimentais e quatro tratamentos. Cada período teve duração de 19 dias, sendo 14 dias para adaptação dos animais às dietas experimentais, e cinco dias para coleta de dados e amostras (fezes, alimentos, sobras dos alimentos, leite, sangue e urina).

\section{Dietas Experimentais}

As dietas foram formuladas para atender às exigências de cabras em lactação, segundo recomendações do NRC (2007). A oferta era feita ad libitum, na forma de mistura completa, em duas refeições diárias logo após as ordenhas às 7 e 15 horas, seguindo a relação volumoso:concentrado de 70:30. Os ingredientes utilizados nas dietas foram o grão de milho moído, farelo de soja, ureia, suplemento vitamínico/mineral, palma forrageira (Nopalea cochenillifera), feno de alfafa (Medicago sativa) e feno de capim Tifton (Cynodon spp.). A composição porcentual e valor nutritivo dos ingredientes e das dietas experimentais encontramse nas Tabelas 1 e 2.

Os fenos foram triturados em máquina forrageira com peneira de crivo de $8 \mathrm{~mm}$, a fim de reduzir a seleção por parte dos animais, e misturados aos demais ingredientes para fornecimento na forma de ração completa. A palma forrageira era triturada diariamente, em máquina própria para processamento de palma.

\section{Coleta de dados e amostras}

Durante o período de adaptação e coletas foram realizadas pesagens diárias da oferta e das sobras de alimentos, de maneira a ser estimado o consumo voluntário em função da sobra referente ao dia anterior, a qual foi controlada em torno de 
$15 \%$ do total de matéria seca (MS) ofertada. A alimentação foi fornecida duas vezes ao dia e o ajuste realizado diariamente. A água foi fornecida ad libitum, sendo o consumo quantificado diariamente, durante o período de coleta de dados, pela relação da oferta e a sobra com a diferença da taxa de evaporação.

Tabela 1. Composição químico-bromatológica dos ingredientes na base da matéria seca.

\begin{tabular}{lccccc}
\hline Item & Milho moído & Farelo de Soja & Palma Forrageira & Feno de Alfafa & $\begin{array}{c}\text { Feno de } \\
\text { Tifton 85 }\end{array}$ \\
\hline Matéria Seca $^{1}$ & 889 & 868 & 83,2 & 826 & 862 \\
Matéria Orgânica $^{2}$ & 984 & 922 & 789 & 902 & 923 \\
Proteína Bruta $^{2}$ & 81,8 & 468 & 75,4 & 180 & 79,1 \\
Extrato Etéreo $^{2}$ & 28,2 & 20,0 & 18,1 & 13,9 & 15,4 \\
FDN $^{2,3}$ & 208 & 185 & 236 & 496 & 756 \\
CNF $^{2,4}$ & 667 & 89,8 & 460 & 213 & 72,2 \\
\hline
\end{tabular}

${ }^{1} \mathrm{~g} / \mathrm{kg}$ do alimento; ${ }^{2} \mathrm{~g} / \mathrm{kg}$ matéria seca; ${ }^{3}$ Fibra em detergente neutro; ${ }^{4}$ Carboidratos não-fibrosos.

Tabela 2. Proporção dos ingredientes e composição químico-bromatológica das dietas experimentais

\begin{tabular}{lcccc}
\hline \multirow{2}{*}{ Ingredientes $(\mathrm{g} / \mathrm{kg})$} & 0 & 33,3 & 66,7 & 100 \\
\cline { 2 - 5 } & 300 & 200 & 100 & 0 \\
Feno de Tifton & 0 & 100 & 200 & 300 \\
Feno de alfafa & 400 & 400 & 400 & 400 \\
Farelo de milho & 190 & 210 & 220 & 240 \\
Farelo de soja & 80 & 65 & 50 & 34 \\
Palma forrageira & 10 & 10 & 10 & 10 \\
Ureia & 10 & 8 & 15 & 10 \\
Núcleo mineral & 3 & 0 & 0 & 0 \\
Calcário calcítico & 7 & 7 & 5 & 6 \\
Fosfato bicálcico & & & & \\
\hline Composição da dieta (g/kg de MS) & 182 & 182 & 182 & 182 \\
\hline Matéria seca (g/kg) & 874 & 876 & 877 & 874 \\
Matéria orgânica & 135 & 139 & 143 & 148 \\
Proteína bruta & 18.8 & 18.9 & 18.8 & 18.9 \\
Extrato etéreo & 375 & 351 & 324 & 299 \\
Fibra em detergente neutro & 352 & 376 & 393 & 416 \\
Carboidratos não-fibrosos & 634 & 605 & 653 & 649 \\
Nutrientes digestíveis totais & & & & \\
\hline
\end{tabular}

A cada período experimental, foram coletadas amostras dos ingredientes das dietas e sobras. A palma forrageira, fenos e as sobras foram imediatamente pré-secas em estufa de ventilação forçada a $55^{\circ} \mathrm{C}$, durante 72 horas. Posteriormente, foram moídas em moinho de facas tipo Willey, passando por peneira de crivo de 2,0 e 1,0 $\mathrm{mm}$, e logo após, acondicionadas em recipientes de polietileno, devidamente identificadas.

\section{Análises bromatológicas das dietas}

As análises bromatológicas das dietas foram realizadas de acordo com os métodos descritos pela AOAC (1990): método de número 934.01 para matéria seca (MS), 930,05 para matéria orgânica (MO) e 981,10 para proteína bruta (PB) e extrato etéreo (EE) método número 920.39. A concentração de FDN foi analisada utilizando uma amilase termoestável e corrigida para cinzas, com base nos procedimentos descritos por Mertens (2002), as amostras foram colocadas em vasos de polietileno com $100 \mathrm{~mL}$ de detergente neutro e autoclavado (Senger et al., 2008). Os carboidratos não fibrosos (CNF) foram calculados segundo Detmann e Valadares Filho (2010). Os nutrientes digestíveis totais (NDT) foram determinados de acordo com Weiss (1999).

\section{Minerais e metabólitos do plasma e urina}

Amostras de sangue foram coletadas por venopunção da veia jugular no $19^{\circ}$ dia de cada período, com tubos vacutainers. As amostras foram imediatamente acondicionadas em ambiente refrigerado, então centrifugadas a $1372 \times g$ por 15 
min. As alíquotas foram colocadas em microtubos de polipropileno tipo ependorffs identificados e armazenados a $-20^{\circ} \mathrm{C}$. Também no último dia de cada período experimental, amostras spot de urina foram coletadas quatro horas após a alimentação, durante micção espontânea. Uma alíquota de 10 $\mathrm{mL}$ de urina foi diluída em $40 \mathrm{~mL}$ de ácido sulfúrico de normalidade 0,036. $\mathrm{O} \mathrm{pH}$ foi mensurado e, caso necessário, foi ajustado para valores inferiores a três, com gotas de ácido sulfúrico concentrado, a fim de evitar a precipitação do ácido úrico, posteriormente armazenadas à $-20^{\circ} \mathrm{C}$.

Foram realizadas análises dos minerais cálcio $(\mathrm{Ca})$, fosforo $(\mathrm{P})$ e magnésio $(\mathrm{Mg})$ no plasma, assim como na urina, e além destes, foram determinadas ureia (Ur) e creatinina (Cr), utilizando-se kits comerciais, em equipamento de espectrofotometria Labmax 24-0. As análises foram feitas no Laboratório de Patologia do Departamento de Medicina Veterinária, da UFRPE.

Foi estimado o volume urinário (VU), multiplicando-se o peso corporal (PC) pela excreção diária de creatinina $(\mathrm{mg} / \mathrm{kg} \mathrm{PC})$, onde se adotou $26,05 \mathrm{mg} / \mathrm{kg}$ PC, segundo Fonseca et al., (2005), e dividiu-se o produto pela concentração de creatinina $(\mathrm{mg} / \mathrm{L})$ na urina spot de cada animal. Foram calculados também a taxa de Depuração Endógena de Creatinina (TDECr) e taxa de excreção de urina (TEU). Foram determinados, os índices de excreção urinários e taxas de excreção fracionadas de $\mathrm{Ca}, \mathrm{P}, \mathrm{Mg}$ e Ur. Os cálculos foram feitos segundo as equações descritas por Garry et al. (1990).

\section{Análise Estatística}

Os dados foram analisados por análise de variância (ANOVA), utilizando o programa Statistical Analysis System (SAS) versão 9.0 (PROC GLM), conforme modelo a seguir: + eijkl

$\mathrm{Y} i j k l=\mu+\mathrm{Q} i+\mathrm{T} j+\mathrm{P}_{k}+(\mathrm{A} / \mathrm{Q}) i l+\mathrm{Q} \times \mathrm{T} i j$

Onde: Yijkl = observação do animal 1 , no período $\mathrm{k}$, submetida ao tratamento $\mathrm{j}$, no quadrado latino i; $\mu$ = média geral, $\mathrm{Qi}=$ efeito do quadrado latino $i$, em que $i=1,2$ e $3 ; \mathrm{Tj}=$ efeito do tratamento $\mathrm{j}$, onde $\mathrm{j}=1,2,3$ e 4 ; (A / Q ) il = efeito do animal 1 , dentro do quadrado latino $\mathrm{i}$, onde $1=1$,
2, 3 e 4; $Q \times$ Tij $=$ efeito da interação entre o quadrado latino $\mathrm{i} \times$ tratamento $\mathrm{j}$; eijkl $=$ erro aleatório associado a cada observação ijkl; eijkl NID (o, s2). Um valor de significância de 0,05 foi escolhido para ser o valor crítico para a probabilidade de erros do tipo I.

\section{Resultados}

Os consumos de MS e MN, expressos em $\mathrm{kg} / \mathrm{dia}$, apresentaram aumento linear $(\mathrm{p}<0,05) \mathrm{em}$ função dos tratamentos. Para cada unidade percentual adicionada do feno de alfafa houve acréscimo de 2,12 g/dia e 12,8 g/dia, respectivamente (Tabela 3 ).

A substituição do feno de Tifton por feno de alfafa provocou aumento linear para o consumo de água ingerida via alimento e consumo de água total $(\mathrm{P}<0,05)$ (Tabela 3). Em relação à quantidade de água ingerida por $\mathrm{kg}$ de MS (Água/kg MS), água/PC\% e água/PC\% e Água/kg $\mathrm{PC}^{0,75}$ não houve influência $(\mathrm{P}>0,05)$ dos tratamentos estudados.

As perdas de água via leite, fezes e urina, assim como a taxa de depuração endógena da creatinina e taxa de excreção de urina não variaram $(\mathrm{P}>0,05)$ entre os tratamentos, com médias de 2,85; 3,18 e $2,90 \mathrm{~L} / \mathrm{dia}$ e $2,78 \mathrm{~mL} / \mathrm{min} / \mathrm{kg} ; 2,02 \mathrm{ml} / \mathrm{min}$ respectivamente.

Os minerais avaliados $\mathrm{Ca}, \mathrm{P} \mathrm{e} \mathrm{Mg}$ no plasma e urina e índices de excreção urinária de cabras alimentadas com feno de alfafa em substituição ao feno de Tifton à base de palma forrageira não houve influência $(\mathrm{P}>0,05)$ das dietas (Tabela 4) para nenhuma das variáveis observadas.

\section{Discussão}

A utilização de palma forrageira em dietas para pequenos ruminantes é relatada por maximizar a ingestão de matéria seca. Esse fato pode ser relacionado à alta palatabilidade, principalmente genótipo palma Miúda, devido primariamente ao alto teor de carboidratos não fibrosos (Ferreira et al., 2009), como amido. A associação de palma com a leguminosa alfafa favoreceu a ingestão da dieta, à medida que substituiu a gramínea Tifton, provavelmente relacionado à diminuição do teor de FDN e melhora do valor nutritivo (Tabela 2), resultando em aumento de taxa de passagem. 
Tabela 3. Balanço hídrico e TDECr de cabras leiteiras alimentadas com feno de alfafa em substituição ao feno de Tifton à base de palma forrageira.

\begin{tabular}{|c|c|c|c|c|c|c|c|}
\hline \multirow{2}{*}{ Variáveis } & \multicolumn{4}{|c|}{ Níveis de substituição (\%) } & \multirow{2}{*}{ EPM } & \multirow{2}{*}{$\mathrm{L}$} & \multirow{2}{*}{ Q } \\
\hline & 0 & 33,3 & 66,7 & 100 & & & \\
\hline CMN (kg/dia) & 12,3 & 13,7 & 13,7 & 13,7 & 0,31 & $0,05^{1}$ & 0,10 \\
\hline CMS (kg/dia) & 2,25 & 2,51 & 2,50 & 2,49 & 0,06 & $0,05^{2}$ & 0,10 \\
\hline \multicolumn{8}{|c|}{ Consumo de água (L/dia) } \\
\hline Alimentação & 10,1 & 11,2 & 11,2 & 11,2 & 0,26 & $0,05^{3}$ & 0,10 \\
\hline Bebida & 0,27 & 0,29 & 0,24 & 0,39 & 0,08 & 0,94 & 0,69 \\
\hline Total & 10,4 & 11,5 & 11,4 & 11,6 & 0,28 & $0,04^{4}$ & 0,15 \\
\hline Água/kg MS & 4,62 & 4,60 & 4,60 & 4,65 & 0,03 & 0,85 & 0,52 \\
\hline Água/PC\% & 22,4 & 24,9 & 25,2 & 26,3 & 0,68 & 0,39 & 0,60 \\
\hline Água/kg PC ${ }^{0,75}$ & 0,58 & 0,65 & 0,65 & 0,68 & 0,02 & 0,21 & 0,41 \\
\hline \multicolumn{8}{|l|}{ Perdas de água (L/dia) } \\
\hline Leite & 2,72 & 2,83 & 2,93 & 2,90 & 0,06 & 0,73 & 0,57 \\
\hline Fezes & 2,83 & 3,72 & 3,11 & 3,06 & 0,16 & 0,08 & 0,10 \\
\hline Urina & 2,98 & 3,45 & 2,68 & 2,52 & 0,27 & 0,83 & 0,54 \\
\hline $\mathrm{TDECr}(\mathrm{ml} / \mathrm{min} / \mathrm{Kg})$ & 2,66 & 2,79 & 2,84 & 2,85 & 0,04 & 0,24 & 0,42 \\
\hline TEU (ml/min) & 2,07 & 2,40 & 1,86 & 1,75 & 0,19 & 0,82 & 0,54 \\
\hline
\end{tabular}

EPM - Erro padrão da média; L - Efeito Linear; Q - Efeito Quadrático; CMN - consumo de matéria natural; CMS - consumo de matéria seca; TDECr - Taxa de depuração endógena da creatinina; TEU - Taxa de excreção de urina;

${ }^{1} \hat{\mathrm{Y}}=12,72+0,012 \mathrm{x} ;{ }^{2} \hat{\mathrm{Y}}=2,331+0,002 \mathrm{x} ;{ }^{3} \hat{\mathrm{Y}}=10,43+0,009 \mathrm{x} ;{ }^{4} \hat{\mathrm{Y}}=10,7+0,010 \mathrm{x}$.

Tabela 4. Ca, P e Mg no plasma e urina e índices de excreção urinária de cabras leiteiras alimentadas com feno de alfafa em substituição ao feno de Tifton a base de palma forrageira.

\begin{tabular}{|c|c|c|c|c|c|c|c|}
\hline \multirow{2}{*}{ Variáveis } & \multicolumn{4}{|c|}{ Níveis de substituição (\%) } & \multirow{2}{*}{ EPM } & \multirow{2}{*}{$\mathrm{L}$} & \multirow{2}{*}{ Q } \\
\hline & 0 & 33,3 & 66,7 & 100 & & & \\
\hline \multicolumn{8}{|l|}{ Plasma } \\
\hline $\mathrm{Ca}(\mathrm{mmol} / \mathrm{L})$ & 1,58 & 1,74 & 1,60 & 1,76 & 0,03 & 0,47 & 0,98 \\
\hline $\mathrm{P}(\mathrm{mmol} / \mathrm{L})$ & 2,47 & 2,54 & 2,26 & 2,39 & 0,09 & 0,91 & 0,87 \\
\hline $\mathrm{Mg}(\mathrm{mmol} / \mathrm{L})$ & 1,58 & 1,51 & 1,46 & 1,50 & 0,04 & 0,68 & 0,45 \\
\hline $\mathrm{Ca}: \mathrm{P}(\mathrm{mmol} / \mathrm{L})$ & 0,69 & 0,73 & 0,76 & 0,81 & 0,03 & 0,83 & 0,91 \\
\hline \multicolumn{8}{|l|}{ Urina } \\
\hline $\mathrm{Ca}(\mathrm{mg} / \mathrm{dL})$ & 0,91 & 0,96 & 1,76 & 1,86 & 0,18 & 0,71 & 0,94 \\
\hline $\mathrm{P}(\mathrm{mg} / \mathrm{dL})$ & 0,78 & 0,17 & 0,83 & 0,48 & 0,23 & 0,96 & 0,75 \\
\hline $\mathrm{Mg}(\mathrm{mg} / \mathrm{dL})$ & 5,71 & 6,14 & 6,25 & 6,46 & 0,15 & 0,48 & 0,71 \\
\hline $\mathrm{Ur}(\mathrm{mg} / \mathrm{dL})$ & 329 & 332 & 343 & 353 & 16,9 & 0,86 & 0,89 \\
\hline \multicolumn{8}{|l|}{ Índices de Excreção } \\
\hline IEUrCa $(\mathrm{mmol} / \mathrm{L})$ & 0,67 & 0,70 & 1,01 & 1,17 & 0,08 & 0,71 & 0,69 \\
\hline IEUrP (mmol/L) & 1,02 & 0,48 & 1,91 & 0,81 & 0,44 & 0,63 & 0,70 \\
\hline IEUrMg $(\mathrm{mmol} / \mathrm{L})$ & 8,96 & 10,25 & 9,26 & 9,34 & 0,55 & 0,55 & 0,50 \\
\hline IEUrUr (mmol/L) & 426 & 483 & 412 & 402 & 21,4 & 0,56 & 0,40 \\
\hline TEFCa $(\%)$ & 0,26 & 0,21 & 0,38 & 0,35 & 0,04 & 0,95 & 0,92 \\
\hline TEFP $(\%)$ & 0,16 & 0,08 & 0,40 & 0,15 & 0,08 & 0,54 & 0,56 \\
\hline TEFMg $(\%)$ & 3,29 & 3,97 & 3,37 & 3,31 & 0,22 & 0,65 & 0,35 \\
\hline TEFUr (\%) & 14,5 & 15,6 & 14,8 & 14,4 & 0,70 & 0,65 & 0,58 \\
\hline
\end{tabular}

EPM - Erro padrão da média; L - Efeito Linear; Q - Efeito Quadrático; Ur - ureia;

O aumento do consumo de água em função da substituição do feno de Tifton por feno de alfafa em dietas à base de palma forrageira pode ser explicado pela ingestão de alimentos (MS e MN) crescente das dietas, visto que o maior aporte da água ingerida pelos animais foi por via alimentar. Silva et al. (2017) relataram que o consumo de água não sofreu efeitos significativos pela substituição do feno de capim Tifton pelo feno de alfafa, para 
ovinos em crescimento, contudo vale ressaltar que as dietas não continham palma forrageira.

O consumo de palma forrageira in natura resulta em redução ingestão voluntária e maior consumo de água total (Costa et al., 2012; Andrade et al., 2016). O que pode ser explicado pelo baixo teor de MS 83,2 g/kg (916,8 g/kg de água) em sua composição (Tabela 1). Resultados semelhantes também foram relatados por Costa et al. (2009) em cabras em lactação, de forma que o aumento dos níveis de palma forrageira diminuiu a necessidade de ingestão de água. Cordova-Torres et al. (2017) descreveram que a restrição de ingestão de água $a d$ libitum em todo o período experimental ( 85 dias) não teve efeito negativo no desempenho de ovinos alimentados com palma forrageira.

Segundo o NRC (2007), seriam necessários 8,38 L/dia para suprir as exigências de água para os animais do presente estudo, contudo, a média encontrada nesta pesquisa foi de 11,26 L/dia, notase que consumo total de água obtido neste trabalho foi muito superior ao preconizado. Destaca-se que as dietas promoveram em média mais de $95 \%$ do total de água consumida pelos animais, suficiente para suprir às exigências desse nutriente sem o fornecimento de água ad libitum, devido à participação da palma forrageira em $400 \mathrm{~g} / \mathrm{kg}$ MS nas dietas.

É relatada diurese associada ao consumo de palma forrageira por caprinos (Vieira et al., 2008; Andrade-Montemayor et al., 2011). A presença de oxalato de cálcio e alta concentração de minerais como potássio podem estar relacionadas ao aumento da excreção urinária (Neto et al. 2016; Galati et al., 2002).

Neste experimento o percentual de matéria seca fecal foi de $20,7 \%$ em média, o que representou $28,2 \%$ da água ingerida, contudo sem diferença associada aos diferentes volumosos avaliados. Apesar de alto conteúdo de água fecal, Andrade et al. (2016) relataram baixo percentual de MS fecal igualmente para animais alimentados com palma forrageira desidratada, o que indicou que não foi a presença de água da palma que provocou fezes aquosas, e sim a sua composição química.

As médias da TDECr $2,7 \mathrm{~mL} / \mathrm{min} / \mathrm{Kg}$ e TEU $2,02 \mathrm{~mL} / \mathrm{min}$, no presente estudo, corroboram com Vieira et al. (2008) em nível de $370 \mathrm{~g} / \mathrm{kg}$ de palma forrageira na dieta de caprinos 2,15 e 1,9, respectivamente.

Apesar da substituição do feno de Tifton por feno de alfafa não ter exercido nenhuma influência significativa nos minerais avaliados $\mathrm{Mg}, \mathrm{Ca}, \mathrm{P}$ no plasma como também nos índices de excreção urinária, os efeitos de dietas à base de palma forrageira, sobre esses parâmetros merecem ser relatados, em função da carência na literatura.

A palma forrageira possui elevados teores de $\mathrm{Ca}$, além de $\mathrm{K}$ e $\mathrm{Mg}$ (Batista et al., 2009). Entretanto, Ben Salem et al. (2002) relataram encontrar $131 \mathrm{~g} / \mathrm{kg}$ MS de oxalato, o que torna reduzida disponibilidade de cálcio, e outros minerais, como resultado do seu sequestro na forma de cristais de oxalato de cálcio (McConn e Nakata, 2004). Está bem estabelecido que o ácido oxálico apresenta grande afinidade com o cálcio e o magnésio, dificultando sua utilização pelo hospedeiro e microrganismos ruminais, de tal maneira, essa questão deve ser considerada durante a formulação de dietas com palma para ruminantes (Bem Salem, 2009).

Andriguetto (1983) acrescentou que no rúmen os oxalatos solúveis podem ser convertidos em carbonatos sem apresentar problemas. Contudo, quando ingestão de oxalato é alta e ultrapassa a capacidade de conversão, os oxalatos solúveis podem ser absorvidos, podendo levar a uma alteração do metabolismo do cálcio e induzir a uma hipocalcemia. No presente estudo, a presença de oxalato não parece ter influenciado o metabolismo do $\mathrm{Mg}$, contudo, diminuiu a absorção do $\mathrm{Ca}$, o que seria uma possível explicação para os baixos valores $(1,67 \mathrm{mmol} / \mathrm{L}$ em média) de $\mathrm{Ca}$ sanguíneo dos animais no presente estudo, visto que os valores de referência são de 2,23-2,93 de Ca total (mmol/L) descritos por Kaneko et al. (2008). Os valores encontrados para $\mathrm{Ca}$ e $\mathrm{P}$ sanguíneos estão semelhantes aos achados de Souto et al. (2013) de cabras com hipocalcemia acometidas com toxemia da prenhez.

Apesar da relação Ca:P estimada das dietas ter sido de 3:1, devido a possível indisponibilidade do cálcio da palma forrageira, desbalanceou a relação Ca:P (mmol/L) sanguínea. Segundo Hansard e Plumlee (1954), quando há uma baixa ingestão de cálcio, há uma maior excreção de fósforo, devido a uma insuficiência de cálcio para a calcificação óssea.

Os índices de excreção de $\mathrm{Mg}, \mathrm{P}$ e ureia corroboram com os valores encontrados por Neto et al. (2016), em dietas com feno Tifton e palma in natura para ovinos em crescimento, contudo diverge do IEUrCa, possivelmente em função dos mesmos fatores descritos anteriormente, para as condições do presente estudo. 


\section{Conclusão}

A substituição do feno de Tifton por feno de alfafa em dietas à base de palma forrageira para cabras em lactação, influencia no consumo de água,, sem alterar os índices de excreção urinária.

\section{Conflito de Interesse}

Os autores declaram não existir conflito de interesse.

\section{Comitê de Ética}

O manejo e os cuidados dos animais foram realizados de acordo com as diretrizes $\mathrm{e}$ recomendações da Comissão de Ética no Uso de Animais (CEUA) da UFRPE, sob o número de licença 56/2015.

\section{Agradecimentos}

Os autores agradecem ao Conselho Nacional de Desenvolvimento Científico e Tecnológico $(\mathrm{CNPq})$ pelo financiamento deste projeto de pesquisa. Agradecemos também à Coordenação de Aperfeiçoamento de Pessoal de Nível Superior (CAPES) pelo subsídio de pesquisa de mestrado.

\section{Referências}

Andrade, S.F.J.D.; Batista, A.M.V.; Carvalho, F.F.R.D.; Lucena, R.B.D.; Andrade, R.D.P.X.D.; Lima Júnior, D.M.D. Fresh or dehydrated spineless cactus in diets for lambs. Acta Scientiarum. Animal Sciences, 38(2): 155-161, 2016.

Andrade-Montemayor, H.M.; Cordova-Torres, A.V.; García-Gasca, T.; Kawas, J.R. Alternative foods for small ruminants in semiarid zones, the case of Mesquite (Prosopis laevigata spp.) and Nopal (Opuntia spp.). Small Ruminant Research, 98(1-3): 83-92, 2011.

Andriguetto, J.M.; Perly, L.; Minardi, I.; Flemming, J.S.; Gemael, A.; Souza, G.A.; Filho, A.B. Nutrição animal, São Paulo: Nobel, 1983. 396p.

Association of Official Analytical Chemists (AOAC). Official methods of analysis, $15^{\text {th }}$ ed. Arlington: AOAC International, 1990.

Batista, A.M.; Ribeiro Neto, A.C.; Lucena, R.B.; Santos, D.C.; Dubeux Jr., J.; Mustafa, A.F. Chemical composition and ruminal degradability of spineless cactus grown in Northeastern Brazil. Rangeland Ecology \& Management, 62(3): 297-301, 2009.
Ben Salem, H.; Nefzaoui, A.; Salem, L.B. Supplementation of Acacia cyanophylla Lindl. foliage-based diets with barley or shrubs from arid areas (Opuntia ficus-indica $\mathrm{f}$. inermis and Atriplex nummularia L.) on growth and digestibility in lambs. Animal Feed Science and Technology, 96(1-2): 1530, 2002.

Ben Salem, H.; Abidi, S. Recent advances on the potential use of Opuntia spp. in livestock feeding. Acta Horticulturae, 811: 317-326, 2009.

Costa, R.G.; Beltrão Filho, E.M.; Medeiros, A.N.; Givisiez, P.E.N.; Egypto, R.D.C.R.; Melo, A.A.S. Effects of increasing levels of cactus pear (Opuntia ficus-indica L. Miller) in the diet of dairy goats and its contribution as a source of water. Small Ruminant Research, 82(1): 62-65, 2009.

Costa, R.G.; Treviño, I.H.; Medeiros, G.R.; Medeiros, A.N.; Pinto, T.F., Oliveira, R.L. Effects of replacing corn with cactus pear (Opuntia ficus indica Mill) on the performance of Santa Inês lambs. Small Ruminant Research, 102(1): 13-17, 2012.

Cordova-Torres, A.V.; Costa, R.G.; Medeiros, A.N.D.; Araújo Filho, J.T.; Ramos, A.O.; Alves, N.D.L. Performance of sheep fed forage cactus with total water restriction. Revista Brasileira de Saúde e Produção Animal, 18(2): 369-377, 2017.

Detmann, E.; Valadares Filho, S.C. On the estimation of non-fibrous carbohydrates in feeds and diets. Arquivo Brasileiro de Medicina Veterinária e Zootecnia, 62(4): 980-984, 2010.

Ferreira, M.A.; Silva, F.M.; Bispo, S.V. Azevedo M. Estratégias na suplementação de vacas leiteiras no semi-árido do Brasil. Revista Brasileira de Zootecnia, 38: 322-329, 2009.

Fonseca, C.E.M.D.; Valadares, R.F.D.; Valadares Filho, S.D.C.; Leão, M.I., Cecon, P.R.; Rodrigues, M.T.; Pina, D.D.S.; Marcondes, M.I.; Paixão, M.L.; Araújo, A. M. Estimativa da produção microbiana em cabras lactantes alimentadas com diferentes teores de proteína na dieta. Revista Brasileira de Zootecnia, 35(3): 1169-1177, 2006.

Galati, E.M.; Tripodo, M.M.; Trovato, A.; Miceli, N.; Monforte, M.T. Biological effect of Opuntia ficus indica (L.) Mill. (Cactaceae) waste matter: Note I: diuretic 
activity. Journal

of

Ethnopharmacology, 79(1): 17-21, 2002.

Garry, F.; Chew, D.J.; Rings, D.M.; Tarr, M.J.; Hoffsis, G.F. Renal excretion of creatinine, electrolytes, protein, and enzymes in healthy sheep. American Journal of Veterinary Research, 51(3): 414-419, 1990.

Hansard, S.L.; Comar, C.L.; Plumlee, M.P. The effects of age upon calcium utilization and maintenance requirements in the bovine. Journal of Animal Science, 13(1): 25-36, 1954.

Kaneko, J.J.; Harvey, J.W.; Bruss, M.L. Clinical biochemistry of domestic animals. California: Academic press, 2008. 928p.

McConn, M.M.; Nakata, P.A. Oxalate reduces calcium availability in the pads of the prickly pear cactus through formation of calcium oxalate crystals. Journal of Agricultural and Food Chemistry, 52: 1371-1374, 2004.

Mertens, D.R. Gravimetric determination of amylase treated neutral detergent fiber in feeds with refluxing in beaker or crucibles: collaborative study. Journal of AOAC International, 85: 1217-1240, 2002.

National Research Council (NRC). Nutrient requirements of small ruminants: sheep, goats, cervids, and New World camelids. Washington, D.C.: National Academy of Science, 2007. 384p.

Neto, J.P.; Soares, P.C.; Batista, A.M.V.; Andrade, S.F.; Andrade, R.P.; Lucena, R.B.; Guim, A. Balanço hídrico e excreção renal de metabólitos em ovinos alimentados com palma forrageira (Nopalea cochenillifera
Salm Dyck). Pesquisa Veterinária Brasileira, 36(4): 322-328, 2016.

Senger, C.C.D.; Kozloski, G.V.; Sanchez, L.M.B.; Mesquita, F.R.; Alves, T.P.; Castagnino, D.S. Evaluation of autoclave procedures for fibre analysis in forage and concentrate feedstuffs. Animal Feed Science and Technology, 146: 169-174, 2008.

Silva, J.C.D.; Véras, A.C.; Carvalho, F.R.D.; Ferreira, M.A.; Souza, E.O.D.; Barreto, L.G.; Lopes, L.A.; Silva, J.V.D. Nutritional value, performance, feeding behavior and serum biochemical profile of sheep fed with alfalfa hay replacing Bermuda grass (Cynodon dactylon (L.) Pers.) hay. Chilean Journal of Agricultural Research, 77(4): 340-345, 2017.

Souto, R.J.; Afonso, J.A.B.; Mendonça, C.L.; Carvalho, C.C.; Silva Filho, A.P.; Cajueiro, J.F.; Lima, E.H.F; Soares, P.C. Achados bioquímicos, eletrolíticos e hormonais de cabras acometidas com toxemia da prenhez. Pesquisa Veterinária Brasileira, 33(10): 1174-1182, 2013.

Vieira, E.L.; Batista, A.M.; Mustafa, A.F.; Araújo, R.F.S.; Soares, P.C.; Ortolane, E.L.; Mori, C.K. Effects of feeding high levels of cactus (Opuntia fícus-indica Mill) cladodes on urinary output and electrolyte excretion in goats. Livestock science, 114(2-3): 354-357, 2008.

Weiss, W.P. Energy prediction equations for ruminant feeds, In: Proceedings of the 61st Cornell nutrition conference for feed manufacturers. New York: Cornell University, 1999. p.176-185. 